\title{
Gene expression dynamics in randomly varying environments
}

\author{
Michael W. Smiley • Stephen R. Proulx
}

Received: 23 October 2008 / Revised: 21 August 2009 / Published online: 16 September 2009 C The Author(s) 2009. This article is published with open access at Springerlink.com

\begin{abstract}
A simple model of gene regulation in response to stochastically changing environmental conditions is developed and analyzed. The model consists of a differential equation driven by a continuous time 2-state Markov process. The density function of the resulting process converges to a beta distribution. We show that the moments converge to their stationary values exponentially in time. Simulations of a two-stage process where protein production depends on mRNA concentrations are also presented demonstrating that protein concentration tracks the environment whenever the rate of protein turnover is larger than the rate of environmental change. Single-celled organisms are therefore expected to have relatively high mRNA and protein turnover rates for genes that respond to environmental fluctuations.
\end{abstract}

Keywords Gene expression · Environmental stochasticity · Stochastic process · Stationary distribution

Mathematics Subject Classification (2000) $92 \mathrm{C} 37 \cdot 92 \mathrm{C} 42 \cdot 60 \mathrm{G} 10$

This paper was greatly improved by the comments of several anonymous reviewers. S. R. Proulx was funded by NSF grant EF-0742582 and by the Baker Center for Bioinformatics at Iowa State University.

M. W. Smiley

Department of Mathematics, Iowa State University,

396 Carver Hall, Ames, IA 50011, USA

e-mail: mwsmiley@iastate.edu

S. R. Proulx ( $\varangle)$

Ecology Evolution and Marine Biology, University of California,

Santa Barbara, Santa Barbara, CA 93106-9620, USA

e-mail: stephen.proulx@gmail.com 


\section{Introduction}

One way that organisms cope with change in the physical and biological environment is by controlling gene expression so that the complement of active proteins can be adjusted (McAdams et al. 2004; Seshasayee et al. 2006; Wittkopp 2007). Clearly, altering protein production to match the environmental demands can be adaptive (Dekel et al. 2005), but the transition from constitutive expression to regulated expression may have its own pitfalls. For example, down-regulating a gene when it is not needed necessarily creates a delay in protein production when the protein is needed in the future.

We consider a cell that encounters a series of environmental conditions that affect gene transcription rates and potentially affect reproductive fitness. The environment alternates between discrete states at random intervals. These discrete states could represent the presence of some metabolite, a signal molecule, or some other aspect of the environment. The environmental state could even represent changes in the physiological or hormonal state that a cell experiences in a multicellular organism. In this paper we use a simple set of assumptions that make our calculations easier and provide a baseline description of gene expression dynamics. In particular, we assume that there are only two environmental states and that the cell has complete information about the state of the environment. Gene expression is regulated in a simple fashion, where the mRNA degradation rates are constant but the rate of transcription varies between environments.

Perhaps the simplest stochastic model of such behavior involves exponential waiting times between environmental shifts. This is a Markovian process, where the history does not influence the future states of the environment. Further, by assuming exponential waiting times there is no additional information available regarding expected waiting time until the next environmental shift. This means that knowing how long the environment has been in one state does not alter the expected waiting time until it shifts to the other state. In particular, this means that the cell has nothing to gain by storing information about the amount of time spent in the current environment. In the simple environment we consider it is unlikely that a more complicated regulatory control structure would be optimal, and so our analysis focusses on simple regulation of transcription rate. However, more realistic environmental scenarios may impose selection for more complex regulatory architectures.

The model we consider is a simple differential equation, $x^{\prime}+\mu x=R(t)$, driven by a continuous time 2-state Markov process $R(t)$, which is sometimes referred to as the telegrapher's process. Stochastic processes of this type have a long history but the literature does not seem to be as well known or as unified as that of diffusion processes, although (Davis 1984) has proposed a general framework for the analysis of "piecewise deterministic Markov processes", which include the type of processes considered here. One of the earliest works seems to be that of McFadden (1959) who consider these in the context of RC circuits and obtained some results on stationary distributions. Further results in the context of RC circuits can be found in Pawula and Rice (1986). More recently (Mazza and Piau 2001) have established a link between Dubins-Freedman Processes and RC filters, and have provided algorithms for computing stationary distributions in a variety of cases. 
As is well-known for the process $R(t)$, the time-dependent density function of the driven process $X(t)$ can be characterized by a generator and a differential equation. However, now the differential equation is a system of partial differential equations. An early work developing the theory of dynamics driven by continuous time finite state Markov processes is that of Milstein and Repin (1972). See also the book by Srinivasan and Vasudevan (1971) and the more recent works (Pinsky 1991; Klyatskin 2005). Although models like the one we consider are primarily found in the mathematics and physics literature, they have recently been used to model gene transcription. For example, in Karmakar and Bose (2004) the telegrapher's process is used to model switches between a gene being in an active or inactive state, with the active state leading to protein synthesis. The same approach is used in Karmakar and Bose (2007) with one of the transition rates protein dependent, and the results compared to a deterministic model that has the cell population density overlapping a bifurcation point in the model.

Several researchers have considered the effects of a stochastically changing environment on cell populations (e.g. Kussell and Leibler 2005; Gander et al. 2007), gene regulation (e.g. Belle et al. 2008; Cook et al. 1998), and protein production (e.g. Karmakar and Bose 2004; Shahrezaei and Swain 2008). In these works the relative rates of cellular processes and environmental switching is shown to be important factor. For example, Bennett and co-workers (Belle et al. 2008) report that in Saccharomyces cerevisiae the metabolic system responds effectively to an environmental stimulus changing at a slow rate, but has virtually no response to the same stimulus changing at a rapid rate. This type of variation in response was observed from a different point of view in simulations of a simple model with switching between active and inactive protein production (Cook et al. 1998). This type of stochastic effect could be caused by variation in chemical reactions, such as changes in the conformation of the promoter, binding of RNA polymerase, and stochastic pausing of RNA polymerase. The simulations showed protein synthesis varied over a wide range when the switching rate was slow but was essentially steady at a rapid rate of switching.

The works of Karmakar and Bose $(2004,2007)$ provide a clearer understanding of how relative rates can determined whether protein synthesis has a unimodal or bimodal distribution. Using a simple activation/deactivation model of protein synthesis they show that bimodal stationary distributions are expected when the rate at which the promoter changes conformation is slow relative to the degradation rates of the protein. This is also our conclusion relative to mRNA synthesis. In addition, we show that all of the moments of the time-dependent distributions converge exponentially to the moments of the stationary distributions, with exponential rates depending on the degradation and switching rates.

We also consider the two step process of transcription and translation from gene to protein and give some heuristic arguments on how the relative rates can effect the cells ability to cope with the environment. Both steps are also considered in Shahrezaei and Swain (2008) from a different modeling approach and with different goals. Starting with a master equation (a large system of ordinary differential equations) for the probabilities of all possible numbers of mRNA and protein molecules, the authors combine a generating function approach with an asymptotic assumption that proteins 
are much more stable the mRNA to reduce the system to one involving only protein probabilities. Again, relative rates play an important role in the analysis.

The outline of the paper is as follows. We begin by introducing basic notation and assumptions about the process $R(t)$, and describe and characterize the time-dependent density of the driven process $X(t)$ in terms of a system of partial differential equations. Stationary distributions, which are Beta distributions, are then explicitly given. With this background developed, we derive time-dependent equations for differences of moments and use them to show the exponential convergence of the moments of the time-dependent process to those of the stationary process. We then extend our model to include the dynamics of protein production and investigate the ability of protein concentrations to track fluctuations in the environment. We argue that the relative rates of environmental fluctuations, mRNA and protein turnover limit the ability of a cell to track its environment. Our simulations indicate that protein concentrations will track changes in the environment provided that both mRNA and protein turnover rates are higher than the rate of environmental change. This suggest that single-celled organisms in variable environments will only persist if mRNA and protein turnover rates are relatively high.

\section{Mathematical framework}

Our model of gene regulation in response to a stochastically changing environment is

$$
\frac{d x}{d t}+\mu x=R(t)
$$

where $x(t)$ represents the expression level (i.e. the concentration) of mRNA, $\mu$ is its degradation rate, and $R(t)$ is the environmental input which is assumed to be a random process switching between the two states $r_{0}$ and $r_{1}$. This corresponds to a system where the gene's enhancer sites can be bound by different transcription factors or cofactors. Once bound, transcription occurs at a constant rate, and the mRNA product breaks down at a constant rate $\mu$. Specifically, $R(t)$ is assumed to be a continuous time 2-state Markov chain, with exponentially distribution waiting times between jumps.

The following notation and standard results, as given in Allen (2003) or Durrett (2004) for example, will be used. The process $R(t)$ can be characterized by its infinitesimal generator

$$
Q=\left[\begin{array}{cc}
-\lambda_{0} & \lambda_{1} \\
\lambda_{0} & -\lambda_{1}
\end{array}\right]
$$

and probability transition matrix $P(t)=\left[p_{i, j}(t)\right]$,

$$
P(t)=\frac{1}{\lambda_{0}+\lambda_{1}}\left[\begin{array}{ll}
\lambda_{1}+\lambda_{0} e^{-\left(\lambda_{0}+\lambda_{1}\right) t} & \lambda_{1}-\lambda_{1} e^{-\left(\lambda_{0}+\lambda_{1}\right) t} \\
\lambda_{0}-\lambda_{0} e^{-\left(\lambda_{0}+\lambda_{1}\right) t} & \lambda_{0}+\lambda_{1} e^{-\left(\lambda_{0}+\lambda_{1}\right) t}
\end{array}\right],
$$

which satisfies the Kolmogorov differential equation $P^{\prime}(t)=Q P(t)$, and the initial condition $P(0)=I$. The entries $\lambda_{0}, \lambda_{1}$ of $Q$ are the transition rates between states, 
and their reciprocals correspond to mean waiting times. Here, we have adopted the convention that $p_{i, j}(t)$ denotes the probability of a transition from state $j$ to state $i$. (This is the transpose, as are $Q$ and $P(t)$, of the usual notational convention but is more convenient in our analysis of stationary distributions for the process $X(t)$. See also Allen (2003) for a consistent use of and argument for this convention.) If the distribution of $R(0)$ is $p(0)=\left[p_{0}(0), p_{1}(0)\right]^{T}$ then the distribution of $R(t)$ is $p(t)=P(t) p(0)$

$$
p(t)=\left[\begin{array}{l}
p_{0}(t) \\
p_{1}(t)
\end{array}\right]=\frac{1}{\lambda_{0}+\lambda_{1}}\left[\begin{array}{l}
\lambda_{1}+\left(\lambda_{0} p_{0}(0)-\lambda_{1} p_{1}(0)\right) e^{-\left(\lambda_{0}+\lambda_{1}\right) t} \\
\lambda_{0}+\left(\lambda_{1} p_{1}(0)-\lambda_{0} p_{0}(0)\right) e^{-\left(\lambda_{0}+\lambda_{1}\right) t}
\end{array}\right] .
$$

The solution of (1) is a continuous time, continuous state stochastic process which we denote by $X(t)$. Assuming $0 \leq r_{0}<r_{1}$, and that $X(0) \in\left[r_{0} / \mu, r_{1} / \mu\right]$ we will have $X(t) \in\left[r_{0} / \mu, r_{1} / \mu\right]$ for $t>0$ (with probability one).

The probability density function $\psi(x, t)$ associated with $X(t)$ can be characterized by a system of two partial differential equations (cf. Srinivasan and Vasudevan 1971; Milstein and Repin 1972; Karmakar and Bose 2004, 2007). Since

$$
\begin{aligned}
\mathcal{P}(X(t) \in[x, x+\Delta x])= & \mathcal{P}\left(X(t) \in[x, x+\Delta x], R(t)=r_{0}\right) \\
& +\mathcal{P}\left(X(t) \in[x, x+\Delta x], R(t)=r_{1}\right)
\end{aligned}
$$

it is natural to consider the cummulative distribution functions $\Psi_{i}(x, t)=\mathcal{P}(X(t) \leq$ $\left.x, R(t)=r_{i}\right),(i=1,2)$, associated with these joint probabilities. The derivatives of these functions with respect to $x$ give the joint density/distribution of $X(t)=x$ and $R(t)=r_{i}$ which will be denoted by $\psi_{i}(x, t)$. Thus $\psi(x, t)$ is given as a marginal density

$$
\psi(x, t)=\psi_{0}(x, t)+\psi_{1}(x, t), \quad x \in\left(\frac{r_{0}}{\mu}, \frac{r_{1}}{\mu}\right), \quad t>0 .
$$

Clearly

$$
\int_{r_{0} / \mu}^{r_{1} / \mu} \psi_{i}(x, t) d x=\mathcal{P}\left(X(t) \in\left[\frac{r_{0}}{\mu}, \frac{r_{1}}{\mu}\right], R(t)=r_{i}\right)=p_{i}(t),
$$

where $\left[p_{0}(t), p_{1}(t)\right]^{T}$ is the distribution of $R(t)$.

Analysis of the transitions between the joint density/distributions of the expressions levels, which result from the transitions between the environmental states $r_{i}$, leads to the characterizing system

$$
\begin{aligned}
\frac{\partial \psi_{0}}{\partial t}+\frac{\partial}{\partial x}\left[\left(r_{0}-\mu x\right) \psi_{0}\right] & =-\lambda_{0} \psi_{0}+\lambda_{1} \psi_{1}, \\
\frac{\partial \psi_{1}}{\partial t}+\frac{\partial}{\partial x}\left[\left(r_{1}-\mu x\right) \psi_{1}\right] & =\lambda_{0} \psi_{0}-\lambda_{1} \psi_{1},
\end{aligned}
$$


for the joint densities $\psi_{0}(x, t), \psi_{1}(x, t)$ on the semi-infinite strip $(x, t) \in\left(r_{0} / \mu\right.$, $\left.r_{1} / \mu\right) \times(0, \infty)$. In addition to $(6)$, the functions should satisfy the integral conditions (5), which formally lead to the appropriate Cauchy data for (6), making it a well-posed hyperbolic system (cf. John 1978). This is seen as follows. Integrating (6) over the interval $\left(r_{0} / \mu, r_{1} / \mu\right)$ results in the identity

$$
\int_{r_{0} / \mu}^{r_{1} / \mu}\left(\left(\psi_{i}\right)_{t}+\left[\left(r_{i}-\mu x\right) \psi_{i}\right]_{x}\right) d x=\frac{d}{d t} \int_{r_{0} / \mu}^{r_{1} / \mu} \psi_{i}(x, t) d x+\left.\left[\left(r_{i}-\mu x\right) \psi_{i}\right]\right|_{r_{0} / \mu} ^{r_{1} / \mu} .
$$

By using (5) we find

$$
\begin{aligned}
& p_{0}^{\prime}(t)+\left.\left[\left(r_{0}-\mu x\right) \psi_{0}\right]\right|_{r_{0} / \mu} ^{r_{1} / \mu}=-\lambda_{0} p_{0}(t)+\lambda_{1} p_{1}(t), \\
& p_{1}^{\prime}(t)+\left.\left[\left(r_{1}-\mu x\right) \psi_{1}\right]\right|_{r_{0} / \mu} ^{r_{1} / \mu}=\lambda_{0} p_{0}(t)-\lambda_{1} p_{1}(t) .
\end{aligned}
$$

Since $p(t)$ satisfies $p^{\prime}(t)=Q p(t)$ the boundary terms must vanish. Hence

$$
\psi_{0}\left(\frac{r_{1}}{\mu}, t\right)=\psi_{1}\left(\frac{r_{0}}{\mu}, t\right)=0, \quad t>0,
$$

while only the weaker conditions

$$
\lim _{x \rightarrow r_{0} / \mu}\left(r_{0}-\mu x\right) \psi_{0}(x, t)=0, \quad \lim _{x \rightarrow r_{1} / \mu}\left(r_{1}-\mu x\right) \psi_{1}(x, t)=0, \quad t>0,
$$

are required at the other end points.

Finally, we note that the characteristic curves for the first equation in (6) trace backward from an arbitrary point $(x, t) \in\left(r_{0} / \mu, r_{1} / \mu\right) \times(0, \infty)$ to either a point on the initial segment where $t=0$ or to a point on the half-line $x=r_{1} / \mu, t>0$; while the characteristic curves for the second equation trace backward to either a point on the initial segment where $t=0$ or to a point on the half-line $x=r_{0} / \mu, t>0$. Thus initial densities $\psi_{0}(x, 0), \psi_{1}(x, 0)$ on $\left(r_{0} / \mu, r_{1} / \mu\right)$ together with the boundary conditions (7) provide the proper Cauchy data for (6). Hence there is a unique solution of (6) together with this Cauchy data.

\section{Stationary distributions}

In our model, the environment changes stochastically over time. We would like to predict the future state of the system and determine whether the levels of gene expression are able to track the environmental change. One way of looking at this is to ask how the system behaves in the long run. In this section, we calculate the environment-specific stationary distributions, as well as the overall stationary distribution. These provide a measure of the relative amount of time that expression will be at any particular level in each environmental state. These can then be used to calculate observable statistics 
of gene expression or plugged into the next level of biological function to determine metabolic function or even fitness.

A stationary distribution $\pi(x)$ of the process (1) is determined by a pair of functions $\pi(x)=\pi_{0}(x)+\pi_{1}(x)$ which are time-independent solutions of the system (6), thus satisfying

$$
\begin{aligned}
& \frac{d}{d x}\left[\left(r_{0}-\mu x\right) \pi_{0}\right]=-\lambda_{0} \pi_{0}+\lambda_{1} \pi_{1}, \\
& \frac{d}{d x}\left[\left(r_{1}-\mu x\right) \pi_{1}\right]=\lambda_{0} \pi_{0}-\lambda_{1} \pi_{1} .
\end{aligned}
$$

The existence and uniqueness of stationary distributions for processes of this type has been well studied (cf. McFadden 1959; Milstein and Repin 1972; Pawula and Rice 1986; Mazza and Piau 2001). Typically Beta distributions are obtained when the waiting times are exponentially distributed as in the present case. See Mazza and Piau (2001) for some results on gamma distributed waiting times.

Although there are many solutions of the system (9) there is only one solution that also satisfies the integral conditions

$$
\int_{r_{0} / \mu}^{r_{1} / \mu} \pi_{0}(x) d x=\frac{\lambda_{1}}{\lambda_{0}+\lambda_{1}}, \int_{r_{0} / \mu}^{r_{1} / \mu} \pi_{1}(x) d x=\frac{\lambda_{0}}{\lambda_{0}+\lambda_{1}}
$$

The values on the right in these equations are the limiting values of the integrals in (5).

Solutions of (9) can be easily found by using the following observation. If $\left[\pi_{0}, \pi_{1}\right]^{T}$ is a solution then by adding the two differential equations we obtain

$$
\frac{d}{d x}\left[\left(r_{0}-\mu x\right) \pi_{0}+\left(r_{1}-\mu x\right) \pi_{1}\right]=0, \quad r_{0} / \mu<x<r_{1} / \mu
$$

This shows that any solution pair of (9) satisfies

$$
\left(r_{0}-\mu x\right) \pi_{0}(x)+\left(r_{1}-\mu x\right) \pi_{1}(x)=K, \quad r_{0} / \mu<x<r_{1} / \mu,
$$

for some constant $K$. Thus $\pi_{1}(x)=\left[\left(\mu x-r_{0}\right) \pi_{0}(x)+K\right] /\left(r_{1}-\mu x\right)$, so that $\pi_{1}$ can be eliminated from the first equation in (9). After solving the resulting equation for $\pi_{0}$, the same relation can then be used to determined $\pi_{1}$.

As can be seen by integrating each of the equations in (9) separately (as was done to obtain (7) and (8)), the solution $\left[\pi_{0}, \pi_{1}\right]^{T}$ satisfying (10) should be one of the solutions with $K=0$. Thus, up to a normalizing factor $C$ it is

$$
\left[\begin{array}{l}
\pi_{0}(x) \\
\pi_{1}(x)
\end{array}\right]=C\left[\begin{array}{l}
\left(\mu x-r_{0}\right)^{-1+\lambda_{0} / \mu}\left(r_{1}-\mu x\right)^{\lambda_{1} / \mu} \\
\left(\mu x-r_{0}\right)^{\lambda_{0} / \mu}\left(r_{1}-\mu x\right)^{-1+\lambda_{1} / \mu}
\end{array}\right] .
$$

It is clear that, although they may be unbounded, both components are integrable functions on $\left(r_{0} / \mu, r_{1} / \mu\right)$. In fact, by using the change of variables $s=\left(\mu x-r_{0}\right) /\left(r_{1}-r_{0}\right)$ 
we have

$$
\int_{r_{0} / \mu}^{r_{1} / \mu}\left(\mu x-r_{0}\right)^{-1+\lambda_{0} / \mu}\left(r_{1}-\mu x\right)^{\lambda_{1} / \mu} d x=\frac{\left(r_{1}-r_{0}\right)^{\left(\lambda_{0}+\lambda_{1}\right) / \mu}}{\mu} B\left(\frac{\lambda_{0}}{\mu}, 1+\frac{\lambda_{1}}{\mu}\right),
$$

where $B(\alpha, \beta)$ is the Beta function (cf. Freund 1971). Similarly

$$
\int_{r_{0} / \mu}^{r_{1} / \mu}\left(\mu x-r_{0}\right)^{\lambda_{0} / \mu}\left(r_{1}-\mu x\right)^{-1+\lambda_{1} / \mu} d x=\frac{\left(r_{1}-r_{0}\right)^{\left(\lambda_{0}+\lambda_{1}\right) / \mu}}{\mu} B\left(1+\frac{\lambda_{0}}{\mu}, \frac{\lambda_{1}}{\mu}\right) .
$$

Since $B(\alpha, \beta)=\Gamma(\alpha) \Gamma(\beta) / \Gamma(\alpha+\beta)$, it follows by a standard property of the Gamma function that

$$
\begin{aligned}
B\left(\frac{\lambda_{0}}{\mu}, 1+\frac{\lambda_{1}}{\mu}\right) & =\frac{\lambda_{1}}{\lambda_{0}+\lambda_{1}} B\left(\frac{\lambda_{0}}{\mu}, \frac{\lambda_{1}}{\mu}\right), \quad B\left(1+\frac{\lambda_{0}}{\mu}, \frac{\lambda_{1}}{\mu}\right) \\
& =\frac{\lambda_{0}}{\lambda_{0}+\lambda_{1}} B\left(\frac{\lambda_{0}}{\mu}, \frac{\lambda_{1}}{\mu}\right) .
\end{aligned}
$$

Therefore, the unique stationary distribution of the process is $\pi=\pi_{0}+\pi_{1}$ where

$$
\begin{aligned}
& \pi_{0}(x)=\frac{\mu\left(\mu x-r_{0}\right)^{-1+\lambda_{0} / \mu}\left(r_{1}-\mu x\right)^{\lambda_{1} / \mu}}{\left(r_{1}-r_{0}\right)^{\left(\lambda_{0}+\lambda_{1}\right) / \mu} B\left(\lambda_{0} / \mu, \lambda_{1} / \mu\right)}, \\
& \pi_{1}(x)=\frac{\mu\left(\mu x-r_{0}\right)^{\lambda_{0} / \mu}\left(r_{1}-\mu x\right)^{-1+\lambda_{1} / \mu}}{\left(r_{1}-r_{0}\right)^{\left(\lambda_{0}+\lambda_{1}\right) / \mu} B\left(\lambda_{0} / \mu, \lambda_{1} / \mu\right)} .
\end{aligned}
$$

\section{Convergence of moments}

In this section, we derive ordinary differential equations for various moments and establish asymptotic estimates showing exponential decay in time. While the previous section shows that the long-run behavior of the system can be calculated, the current section shows how quickly that long run behavior is approached. Because we find that the stationary distribution is approached as an exponential decay function that depends on the rate parameters, we can infer that the stationary distribution is reasonable measure of the behavior when organisms persist over several alternations of the environment.

Let $\psi_{0}, \psi_{1}$ be the solutions of (6) satisfying (5), and $\pi_{0}, \pi_{1}$ be the unique stationary distributions satisfying (9) and (10) given in (13). We consider time varying moments of the differences

$$
M_{i, k}(t)=\int_{r_{0} / \mu}^{r_{1} / \mu} x^{k}\left(\psi_{i}(x, t)-\pi_{i}(x)\right) d x, \quad i=0,1 .
$$


The moments $M_{0,0}(t), M_{1,0}(t)$ are actually already known. From (2), (5), and (10), it follows that

$$
\left[\begin{array}{l}
M_{0,0}(t) \\
M_{1,0}(t)
\end{array}\right]=\left[\begin{array}{l}
p_{0}(t) \\
p_{1}(t)
\end{array}\right]-\frac{1}{\lambda_{0}+\lambda_{1}}\left[\begin{array}{l}
\lambda_{1} \\
\lambda_{0}
\end{array}\right]=e^{-\left(\lambda_{0}+\lambda_{1}\right) t}\left[\begin{array}{l}
M_{0,0}(0) \\
M_{1,0}(0)
\end{array}\right]
$$

Taking the difference of first equation in (6) and the first equation in (9) we find

$$
\begin{aligned}
M_{0, k}^{\prime}(t) & =\int_{r_{0} / \mu}^{r_{1} / \mu} x^{k} \frac{\partial \psi_{i}}{\partial t}(x, t) d x \\
& =\int_{r_{0} / \mu}^{r_{1} / \mu} x^{k}\left(\frac{\partial}{\partial x}\left(\left(\mu x-r_{0}\right)\left(\psi_{0}-\pi_{0}\right)\right)-\lambda_{0}\left(\psi_{0}-\pi_{0}\right)+\lambda_{1}\left(\psi_{1}-\pi_{1}\right)\right) d x .
\end{aligned}
$$

Using (7) and (8) and the explicit form of $\pi_{0}$ it follows that

$$
\begin{aligned}
& \int_{r_{0} / \mu}^{r_{1} / \mu} x^{k}\left(\left(\mu x-r_{0}\right)\left(\psi_{0}-\pi_{0}\right)\right)_{x} d x \\
& =\left.x^{k}\left(\left(\mu x-r_{0}\right)\left(\psi_{0}-\pi_{0}\right)\right)\right|_{r_{0} / \mu} ^{r_{1} / \mu}-\int_{r_{0} / \mu}^{r_{1} / \mu} k x^{k-1}\left(\left(\mu x-r_{0}\right)\left(\psi_{0}-\pi_{0}\right)\right) d x \\
& =-\mu k \int_{r_{0} / \mu}^{r_{1} / \mu} x^{k}\left(\psi_{0}-\pi_{0}\right) d x+r_{0} \int_{r_{0} / \mu}^{r_{1} / \mu} x^{k-1}\left(\psi_{0}-\pi_{0}\right) d x,
\end{aligned}
$$

for any $k \geq 1$. Thus, for $k \geq 1$, the moment $M_{0, k}(t)$ satisfies

$$
M_{0, k}^{\prime}(t)=-\left(\mu k+\lambda_{0}\right) M_{0, k}(t)+\lambda_{1} M_{1, k}(t)+r_{0} k M_{0, k-1}(t) .
$$

A similar calculation applies to $M_{1, k}(t)$ showing that for $k \geq 1$ the $k$-th moments satisfy the linear nonhomogeneous system

$$
\left[\begin{array}{l}
M_{0, k} \\
M_{1, k}
\end{array}\right]^{\prime}=\left[\begin{array}{cc}
-\left(\mu k+\lambda_{0}\right) & \lambda_{1} \\
\lambda_{0} & -\left(\mu k+\lambda_{1}\right)
\end{array}\right]\left[\begin{array}{l}
M_{0, k} \\
M_{1, k}
\end{array}\right]+k\left[\begin{array}{l}
r_{0} M_{0, k-1} \\
r_{1} M_{1, k-1}
\end{array}\right]
$$

In matrix-vector notation we have $M_{k}^{\prime}=(-\mu k I+Q) M_{k}+D_{k} M_{k-1}$ where $M_{k}(t)=$ $\left[M_{0, k}(t), M_{1, k}(t)\right]^{T}, Q$ is the infinitesimal generator for the process $R(t)$, and $D_{k}=\operatorname{diag}\left[r_{0} k, r_{1} k\right]$ is a diagonal matrix. Since $\exp (t Q)=P(t)$ it follows that 
$\exp (t(-\mu k I+Q))=e^{-\mu k t} P(t)$. Hence the variation of parameters representation of the solution gives

$$
M_{k}(t)=e^{-\mu k t} P(t) M_{k}(0)+\int_{0}^{t} e^{-\mu k(t-s)} P(t-s) D_{k} M_{k-1}(s) d s .
$$

Let $\|v\|_{1}=\left|v_{1}\right|+\left|v_{2}\right|$ denote that standard 1-norm of a vector $v=\left[v_{1}, v_{2}\right]^{T}$, and $\|A\|_{1}$ the induced matrix norm (cf. Horn and Johnson 1985). This choice of norm is convenient since the induced matrix norm turns our to be the maximum of all column sums of the matrix. Thus $\|P(t)\|_{1}=1$, for all $t$, and $\left\|D_{k}\right\|_{1}=r_{1} k$. Hence, for all $k \geq 1$,

$$
\left\|M_{k}(t)\right\|_{1} \leq e^{-\mu k t}\left\|M_{k}(0)\right\|_{1}+r_{1} k \int_{0}^{t} e^{-\mu k(t-s)}\left\|M_{k-1}(s)\right\|_{1} d s .
$$

Clearly estimates on $\left\|M_{k-1}(t)\right\|_{1}$ lead to estimates on $\left\|M_{k}(t)\right\|_{1}$. Since we know $\left\|M_{0}(t)\right\|_{1} \leq\left\|M_{0}(0)\right\|_{1} \exp \left(-\left(\lambda_{0}+\lambda_{1}\right) t\right)$ this leads inductively to estimates on all of the moments.

Let $\left\{h_{k}(t)\right\}_{k=0}^{\infty}$ be the sequence of functions defined inductively by

$$
h_{0}(t)=e^{-\left(\lambda_{0}+\lambda_{1}\right) t}, \quad \text { and } \quad h_{k}(t)=e^{-\mu k t} \int_{0}^{t} e^{\mu k s} h_{k-1}(s) d s, \quad k \geq 1
$$

We show by induction that

$$
\left\|M_{k}(t)\right\|_{1} \leq \sum_{j=1}^{k}\left(\begin{array}{l}
k \\
j
\end{array}\right)\left\|M_{j}(0)\right\|_{1}\left(\frac{r_{1}}{\mu}\right)^{k-j} e^{-\mu j t}+k ! r_{1}^{k}\left\|M_{0}(0)\right\|_{1} h_{k}(t) .
$$

This is easily seen to hold when $k=1$.

Suppose (16) is valid with $k$ replaced by $k-1$. Then the second term in (15) satisfies

$$
r_{1} k \int_{0}^{t} e^{-\mu k(t-s)}\left\|M_{k-1}(s)\right\|_{1} d s \leq r_{1} k e^{-\mu k t}\left(I_{1}(t)+I_{2}(t)\right),
$$

where

$$
\begin{aligned}
I_{1}(t) & =\sum_{j=1}^{k-1}\left(\begin{array}{c}
k-1 \\
j
\end{array}\right)\left\|M_{j}(0)\right\|_{1}\left(\frac{r_{1}}{\mu}\right)^{k-j-1} \int_{0}^{t} e^{\mu(k-j) s} d s \\
& =\sum_{j=1}^{k-1}\left(\begin{array}{c}
k-1 \\
j
\end{array}\right)\left\|M_{j}(0)\right\|_{1}\left(\frac{r_{1}}{\mu}\right)^{k-j-1} \frac{e^{\mu(k-j) t}-1}{\mu(k-j)}
\end{aligned}
$$


and

$$
I_{2}(t)=(k-1) ! r_{1}^{k-1}\left\|M_{0}(0)\right\|_{1} \int_{0}^{t} e^{\mu k s} h_{k-1}(s) d s .
$$

Clearly $r_{1} k e^{-\mu k t} I_{2}(t)=k ! r_{1}^{k}\left\|M_{0}(0)\right\|_{1} h_{k}(t)$, and since $e^{\mu(k-j) t}-1 \leq e^{\mu(k-j) t}$

$$
r_{1} k e^{-\mu k t} I_{1}(t) \leq \sum_{j=1}^{k-1}\left(\begin{array}{l}
k \\
j
\end{array}\right)\left\|M_{j}(0)\right\|_{1}\left(\frac{r_{1}}{\mu}\right)^{k-j} e^{-\mu j t}
$$

Inequality (16) follows directly by adding the first term in (15) to these upper bounds.

We now show each of the functions $h_{k}(t)$ decays exponentially to zero as $t \rightarrow \infty$. The cases $\lambda_{0}+\lambda_{1}<\mu$ and $\lambda_{0}+\lambda_{1} \geq \mu$ are considered separately. First we assume $\lambda_{0}+\lambda_{1} \geq \mu$. Then $h_{0}(t)=\exp \left(-\left(\lambda_{0}+\lambda_{1}\right) t\right) \leq e^{-\mu t}, t \geq 0$, and hence

$$
h_{1}(t)=e^{-\mu t} \int_{0}^{t} e^{\mu s} h_{0}(s) d s \leq t e^{-\mu t} .
$$

An easy induction argument then shows

$$
h_{k}(t) \leq \frac{t e^{-\mu t}}{\mu^{k-1}(k-1) !} t \geq 0, \quad \text { when } \lambda_{0}+\lambda_{1} \geq \mu .
$$

Next we assume $\lambda_{0}+\lambda_{1}<\mu$. For notational convenience we set $\lambda=\lambda_{0}+\lambda_{1}$, so that $h_{0}(t)=e^{-\lambda t}$. By the mean value theorem applied to $f(x)=e^{-x t}$ we find

$$
h_{1}(t)=e^{-\mu t} \int_{0}^{t} e^{\mu s} e^{-\lambda s} d s=\frac{e^{-\lambda t}-e^{-\mu t}}{\mu-\lambda}=t e^{-\theta t} \leq t e^{-\lambda t}, \quad t \geq 0 .
$$

By induction it follows that for $k \geq 2$

$$
h_{k}(t) \leq \frac{t e^{-\lambda t}}{(2 \mu-\lambda) \cdots(k \mu-\lambda)}=\frac{t e^{-\lambda t}}{\prod_{i=2}^{k}(i \mu-\lambda)}
$$

Since $i \mu-\lambda=(i-1) \mu+(\mu-\lambda)>(i-1) \mu$ it then follows that

$$
h_{k}(t) \leq \frac{t e^{-\lambda t}}{\mu^{k-1}(k-1) !}, \quad t \geq 0, \quad \text { when } \lambda=\lambda_{0}+\lambda_{1}<\mu .
$$

These two cases can be combined to show

$$
k ! r_{1}^{k} h_{k}(t) \leq k\left(\frac{r_{1}}{\mu}\right)^{k} \mu t \exp \left(-\min \left\{\mu, \lambda_{0}+\lambda_{1}\right\} t\right), \quad t \geq 0 .
$$


It is now clear from (16) that each of the moments $M_{i, k}(t)$ decays exponentially to zero, as $t \rightarrow \infty$.

\section{Convergence of statistics}

Biological function is only indirectly dependent on gene expression. The ultimate function will depend on protein production and the activity of those proteins and their products. Calculating this downstream functionality will often require computing expectations of functions of gene expression. In this section, we show that such statistics also converge exponentially in time.

The convergence of moments shown in the previous section leads to the convergence of all statistics associated with the process $X(t)$. Since the density function $\psi(x, t)$ associated with the process $X(t)$, as given in (4), is the sum of $\psi_{0}(x, t)$ and $\psi_{1}(x, t)$ and since all of the moments of $\psi_{i}$ converge to the moments of $\pi_{i}$ $(i=0,1)$, it follows that the moments of $\psi(x, t)$ converge to the moments of the sum $\pi(x)=\pi_{0}(x)+\pi_{1}(x)$, as $t \rightarrow \infty$.

According to (13) the stationary distribution $\pi=\pi_{0}+\pi_{1}$ is explicitly given by

$$
\pi(x)=\frac{\mu\left(r_{1}-r_{0}\right)}{\left(r_{1}-r_{0}\right)^{\left(\lambda_{0}+\lambda_{1}\right) / \mu} B\left(\frac{\lambda_{0}}{\mu}, \frac{\lambda_{1}}{\mu}\right)}\left(\mu x-r_{0}\right)^{\lambda_{0} / \mu-1}\left(r_{1}-\mu x\right)^{\lambda_{1} / \mu-1} .
$$

This is shown to be a beta distribution on $\left(r_{0} / \mu, r_{1} / \mu\right)$, with parameters $\alpha=\lambda_{0} / \mu$ and $\beta=\lambda_{1} / \mu$, as follows.

The standard beta distribution $\rho(s)$, with parameters $\alpha$ and $\beta$, is defined by

$$
\rho(s)=\frac{1}{B(\alpha, \beta)} s^{\alpha-1}(1-s)^{\beta-1}, \quad 0<s<1
$$

with $\rho(s)=0$ elsewhere. If $S$ is a random variable with this distribution, then by a change of variables (cf. Freund 1971) $X=r_{0} / \mu+S\left(r_{1}-r_{0}\right) / \mu$ is a random variable with the distribution

$$
\pi(x)=\frac{\mu}{r_{1}-r_{0}} \rho\left(\frac{\mu x-r_{0}}{r_{1}-r_{0}}\right)=\frac{\mu}{\left(r_{1}-r_{0}\right) B(\alpha, \beta)}\left(\frac{\mu x-r_{0}}{r_{1}-r_{0}}\right)^{\alpha-1}\left(\frac{r_{1}-\mu x}{r_{1}-r_{0}}\right)^{\beta-1} .
$$

Setting $\alpha=\lambda_{0} / \mu$ and $\beta=\lambda_{1} / \mu$, we obtain (17).

Since all of the moments converge it follows (see Athreya and Lahiri (2006) for the discrete case) that all of the statistics of the process $X(t)$ converge to the statistics of a random variable with the distribution $\pi(x)$. That is, for any bounded continuous function $f(x)$ defined on $\mathbb{R}$ we have

$$
\lim _{t \rightarrow \infty} \int_{r_{0} / \mu}^{r_{1} / \mu} f(x) \psi(x, t) d x=\int_{r_{0} / \mu}^{r_{1} / \mu} f(x) \pi(x) d x
$$


or in terms of expected values $\lim _{t \rightarrow \infty} E(f(X(t)))=E\left(f\left(X_{\infty}\right)\right)$, where $X_{\infty}$ is a random variable with the beta distribution $\pi(x)$. We remark that in this setting a proof can be based on the density of polynomials in the uniform norm on compact subintervals of $\mathbb{R}$ (i.e. Weierstrass's approximation theorem).

It is easy to see that the graph of the distribution $\pi(x)$, like all beta distributions, may have a variety of shapes. For example, if $\lambda_{0} / \mu<1$ then $x=r_{0} / \mu$ is a vertical asymptote, while if $\lambda_{0} / \mu>1$ the graph passes through the point $\left(r_{0} / \mu, 0\right)$ on the $x$-axis. Thus, depending on the ratios of the transition rates (i.e. mean waiting times) to the turnover rate $\mu$, the distribution could be sharply peaked within the interval or have most of its mass near the end points, among many other alternatives.

\section{Two-stage model of mRNA and protein concentration}

Our analysis has relied on a one-step model where an mRNA gene product is produced at an environment dependent rate and decays at a fixed rate. Fitness must, in the end, depend on the protein complement of a cell and the internal structural and physiological conditions created by those proteins. To address this, we consider a two stage model where protein production depends on the concentration of mRNA. In our current model we simply assume that the rate of protein production is proportional to mRNA concentration and that the rate of protein decay is fixed. This excludes several known biologically important processes including posttranscriptional regulation (Halbeisen et al. 2008), diminishing returns in translation due to ribosome saturation, and targeted degradation of protein. This leads to a modified system

$$
\begin{aligned}
& \frac{d x}{d t}+\mu_{r} x=R(t) \\
& \frac{d y}{d t}+\mu_{p} y=r_{p} x,
\end{aligned}
$$

where $\mu_{r}$ and $\mu_{p}$ are the respective decay rates of mRNA and protein, $r_{p}$ is the environment independent rate at which protein is translated from active mRNA, and $R(t)$ is the continuous-time 2-state Markov process modeling the switching environmental conditions as before. This model gives rise to a joint stochastic process $(X(t), Y(t))$ for the levels of mRNA and protein. As with the single variable process the stationary probability density function $\rho(x, y)$ for the joint process can be written in the form $\rho(x, y)=\rho_{0}(x, y)+\rho_{1}(x, y)$, with $\rho_{0}, \rho_{1}$ now characterized by the first order system of partial differential equations

$$
\begin{aligned}
\frac{\partial}{\partial x}\left[\left(r_{0}-\mu_{r} x\right) \rho_{0}\right]+\frac{\partial}{\partial y}\left[\left(r_{p} x-\mu_{p} y\right) \rho_{0}\right] & =-\lambda_{0} \rho_{0}+\lambda_{1} \rho_{1}, \\
\frac{\partial}{\partial x}\left[\left(r_{1}-\mu_{r} x\right) \rho_{1}\right]+\frac{\partial}{\partial y}\left[\left(r_{p} x-\mu_{p} y\right) \rho_{1}\right] & =\lambda_{0} \rho_{0}-\lambda_{1} \rho_{1},
\end{aligned}
$$


along with the integral identities

$$
\int_{\mathcal{R}} \rho_{0}(x, y) d A=\frac{\lambda_{1}}{\lambda_{0}+\lambda_{1}}, \quad \int_{\mathcal{R}} \rho_{1}(x, y) d A=\frac{\lambda_{0}}{\lambda_{0}+\lambda_{1}},
$$

where $\mathcal{R}=[a, b] \times[c, d]$, with $a=r_{0} / \mu_{r}, b=r_{1} / \mu_{r}, c=r_{p} a / \mu_{p}$, and $d=$ $r_{p} b / \mu_{p}$.

The PDE (18) is a symmetric hyperbolic system subject to integral side conditions. Little seems to be known theoretically for problems of this type, and our efforts to find analytical solutions have not been successful. We have therefore used stochastic simulations to obtain approximations of the density $\rho(x, y)$.

We consider a discrete version of the joint stochastic process defined as follows. Assuming the process starts at $\left(x_{0}, y_{0}\right)$ with the environment in state $r_{0}$, we find that as long as the environment remains in this state the dynamics determine the state at time $t$ to be

$$
\left[\begin{array}{l}
x(t) \\
y(t)
\end{array}\right]=\frac{1}{\mu_{p}-\mu_{r}}\left[\begin{array}{cc}
\left(\mu_{p}-\mu_{r}\right) e^{-\mu_{r} t} & 0 \\
r_{p}\left(e^{-\mu_{r} t}-e^{-\mu_{p} t}\right) & \left(\mu_{p}-\mu_{r}\right) e^{-\mu_{p} t}
\end{array}\right]\left[\begin{array}{l}
x_{0}-a \\
y_{0}-c
\end{array}\right]+\left[\begin{array}{l}
b \\
d
\end{array}\right]
$$

If $t$ is drawn from the distribution $\operatorname{Exp}\left(\lambda_{0}\right)$ of waiting times before the environment switches, this determines a random mapping on $\mathcal{R}$. If the environment starts in state $r_{1}$ then $x(t), y(t)$ are given by the same expression as above, but with the roles of $a$ (resp. $c$ ) and $b$ (resp. $d$ ) interchanged. Again a random map on $\mathcal{R}$ is defined by drawing from the distribution $\operatorname{Exp}\left(\lambda_{1}\right)$ of waiting times before the environment switches. This gives a pair of random maps on $\mathcal{R}$ of the form

$$
F_{i, t}(z)=A(t) z+b_{i}(t), \quad z \in \mathcal{R}
$$

where $A(t)$ is a $(2 \times 2)$ matrix depending only on $\mu_{r}, \mu_{p}, r_{p}$, with $t$ being randomly drawn from $\operatorname{Exp}\left(\lambda_{i}\right)$, and $b_{i}(t)$ being a column vector depending on the state $r_{i}$ of the environment and the matrix $A(t)$. This defines a discrete process associated with the continuous process $(X(t), Y(t))$ that is given in terms of iterated random functions (Diaconis and Freedman 1999). This process consists of points in $\mathcal{R}$ at which the flow of the dynamical system reverses due to an environmental switch. Although the choice of the index $i$ is deterministic, since it is alternatively taken as 0 or 1 , it has been shown in the 1-dimensional case that there are distributions of waiting times such that the stationary distribution of this process follows the same stationary distribution as the process in which $i$ is chosen randomly (Mazza and Piau 2001).

Let $\left\{Z_{k}\right\}_{k=1}^{\infty}$ denote the discrete process generated by iteration of the random functions described above. For simplicity we assume that the distributions of waiting times in the two environments are the same, so that in the stationary regime the fraction of time spend in each environment is the same. Operating under the assumption that (here $\delta_{z}(A)=1$ if $z \in A$ and is zero otherwise) 


$$
\frac{1}{N} \sum_{k=1}^{N} \delta_{Z_{k}}
$$

converges to the distribution of the process (cf. Diaconis and Freedman 1999), with convergence in an appropriate sense, we use stochastic simulations to compute realizations of $\left\{Z_{k}\right\}_{k=1}^{N}$, for large $N$ and then an approximation $\rho_{N}(x, y)$ of the density $\rho(x, y)$ as follows. Let $[a, b]$ be subdivided into $M_{x}$ subintervals of equal width $\Delta x$, and $[c, d]$ be subdivided into $M_{y}$ subintervals of equal width $\Delta y$. This determines $M_{x} M_{y}$ subrectangles $R_{i, j}$ of $\mathcal{R}$. We define $\rho_{N}(x, y)$ as a piecewise constant function, with $\rho_{N}(x, y)=\rho_{i, j}$, for all $(x, y) \in R_{i, j}$, where

$$
\rho_{i, j} \Delta x \Delta y=\frac{1}{N} \sum_{k=1}^{N} \delta_{Z_{k}}\left(R_{i, j}\right)
$$

Piecewise constant marginal densities are then determined by $\sigma_{N}^{y}(x)=\sigma_{i}^{y}$ on $\left[x_{i-1}, x_{i}\right], \sigma_{N}^{x}(y)=\sigma_{j}^{x}$ on $\left[y_{j-1}, y_{j}\right]$, with

$$
\sigma_{i}^{y}=\sum_{j=1}^{M_{y}} \rho_{i, j} \Delta y, \quad i=1, \ldots, M_{x}, \quad \sigma_{j}^{x}=\sum_{i=1}^{M_{x}} \rho_{i, j} \Delta x, \quad j=1, \ldots, M_{y} .
$$

One of the marginal densities of $\rho(x, y)$ can be computed analytically. Integrating the system (18) with respect to $y$ over the interval $[c, d]$, and using the fact $\rho_{0}, \rho_{1}$ should be zero on the edges $y=c, y=d$ (a consequence of the integral identities $\rho_{0}, \rho_{1}$ must satisfy), leads to the conclusion that

$$
\pi_{0}(x)=\int_{c}^{d} \rho_{0}(x, y) d y, \quad \pi_{1}(x)=\int_{c}^{d} \rho_{1}(x, y) d y,
$$

where $\pi_{0}, \pi_{1}$ are the densities satisfying (9) and (10). Thus the marginal density $\sigma^{y}(x)$ of $x$ is $\pi(x)$, the stationary density of the single equation given in (17). Since the moments of $\pi$ are known, moments of its simulated approximation $\sigma_{N}^{y}$ can be compared to them for an indication of the validity of the methods being used.

Our main interest in modeling the two-stage process is to determine when protein levels can effectively track the changing environment. The single-stage model showed that there is a qualitative change in the stationary distribution such that there is a modal value when mRNA turnover is faster than the rate of environmental change. In the two-stage model, the translation system experiences a continuously changing "environment" defined by the current concentration of mRNA. Thus, it is intuitively clear that protein distributions can only track the environment if mRNA concentration transmits the environmental signal. Complementary to this principle, mRNA concentration will never fluctuate with a period shorter than that of the environmental change, so protein turnover does not need to be quick relative to mRNA turnover, but it does need to be quick relative to the rate of environmental change. 
A The joint density of mRNA and protein

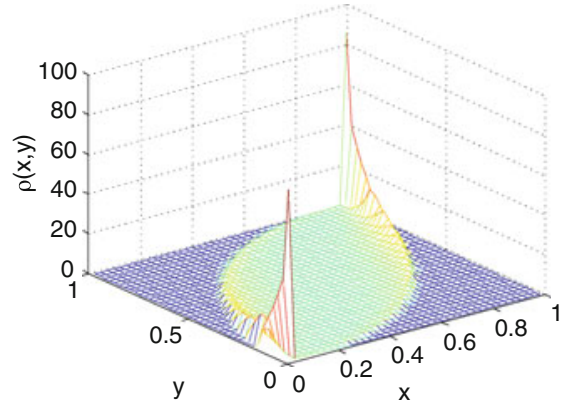

B The joint density of mRNA and protein

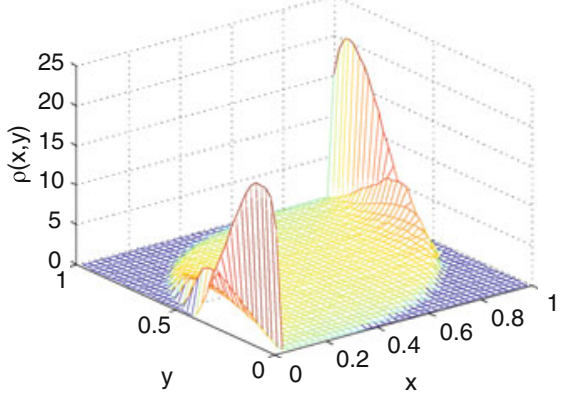

Fig. 1 The joint density functions for mRNA $(x)$ and protein $(y)$. For both panels, parameters are $r_{0}=0$, $r_{1}=1, \mu_{r}=0.025, r_{p}=0.01, \lambda_{0}=\lambda_{1}=0.01$. Panel A shows a case where protein turnover is relatively high $\left(\mu_{p}=0.2\right)$ while in panel $\mathrm{B}$ protein turnover is slower than the rate of environmental switching $\left(\mu_{p}=0.0085\right)$. In both figures, there are large peaks around $x=0$ and $x=1$, indicating that mRNA densities track the environment. A curved ridge connects these peaks and reveals the trace of the joint densities during the transition between environments. This curved ridge shows that protein density achieves less proportional change at any given point than mRNA (i.e., protein density changes more slowly than mRNA density). In panel B, this effect is more pronounced and the peaks are more spread out in the $y$ direction, indicating that protein density does not track environmental change well

To demonstrate this effect, we simulated cases in which $\mu_{p}$ was greater than or less than $\lambda=\lambda_{0}=\lambda_{1}$ (see supplemental file for Matlab simulation code). In all of the simulations reported, we set $r_{0}=0, r_{1}=1, \mu_{r}=0.025, r_{p}=0.01, \lambda_{0}=\lambda_{1}=0.01$, and used $N=500,000$ simulated realizations. Plots were normalized to the unit interval or unit square respectively. The expected value and variance of the exact marginal distribution $\pi$ were therefore 0.5 and (rounded to 4 digits) 0.1389 . The expected values (rounded to 4 digits) of the simulated marginal densities $\sigma_{N}^{y}$ were all in the interval $(0.4998,0.501)$, and the variances (also rounded) were in the interval $(0.1372,0.1374)$, and hence showed very good agreement with those of $\pi$.

Figure 1 shows the range of qualitative behaviors as seen in the joint stationary distribution. So long as both $\mu_{r}, \mu_{p}>\lambda$ the joint distribution has most of its density near the corner points (Fig. 1a). This is also reflected in the marginal distributions shown in Fig. 2a. When protein turnover is low while mRNA turnover is high, relative to the environmental switching rate, the joint density is seen to smear out along the vertical edges of $\mathcal{R}$ (Fig. 1b). The marginal density of mRNA remains unchanged but the marginal density of protein density becomes unimodal with its mode at intermediate protein concentrations (Fig. 2b). Figure 3 shows that this qualitative change takes place as the decay rate $\mu_{p}$ crosses through a small neighborhood of the switching rate $\lambda$. Figure 3 also shows that the marginal protein density appears to have 3 modes as this transition takes place.

\section{Discussion}

We have analyzed a model of gene expression in response to environmental fluctuations. Our main finding is that the probability density of gene expression approaches 

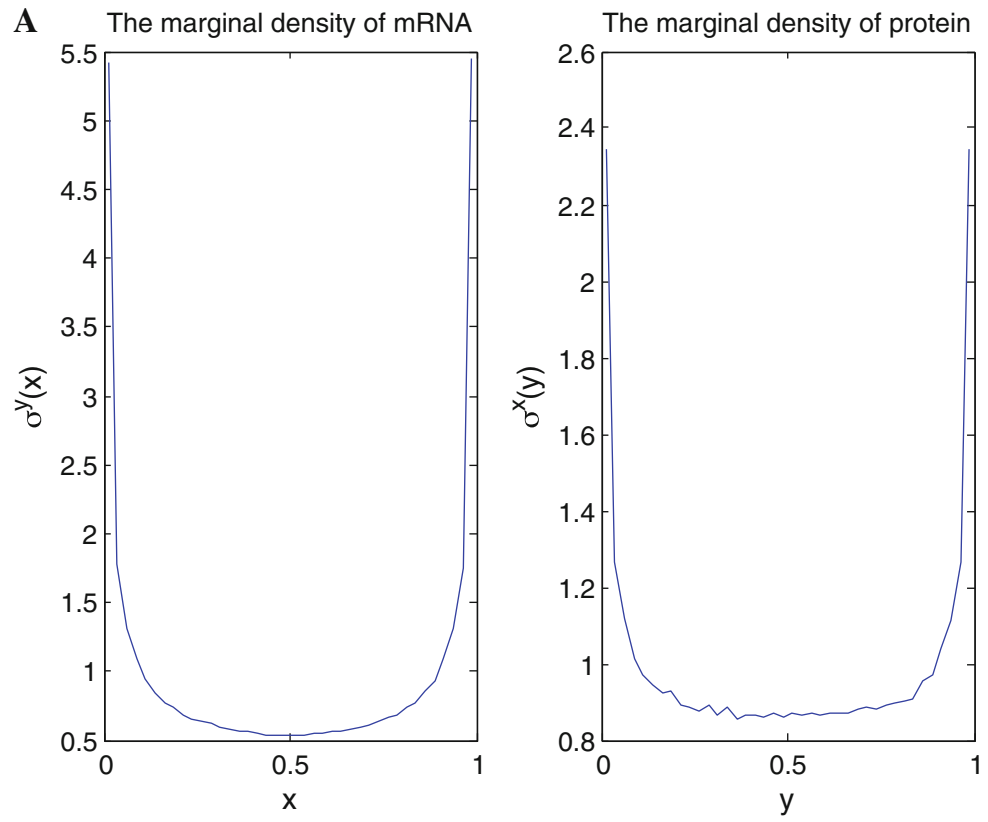

B The marginal density of mRNA

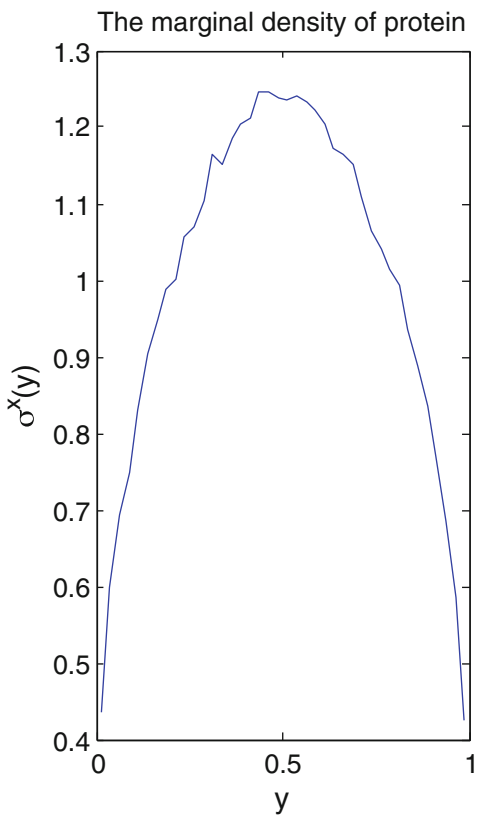

Fig. 2 The marginal density functions for mRNA $(x)$ and protein $(y)$. The parameters are set as in Fig. 1. In panel A $\mu_{p}=0.2$ while in panel B $\mu_{p}=0.0085$. In panel A, protein distributions are highly peaked at $y=0$ and $y=1$, while panel $\mathrm{B}$ shows a strong mode in the protein distribution 

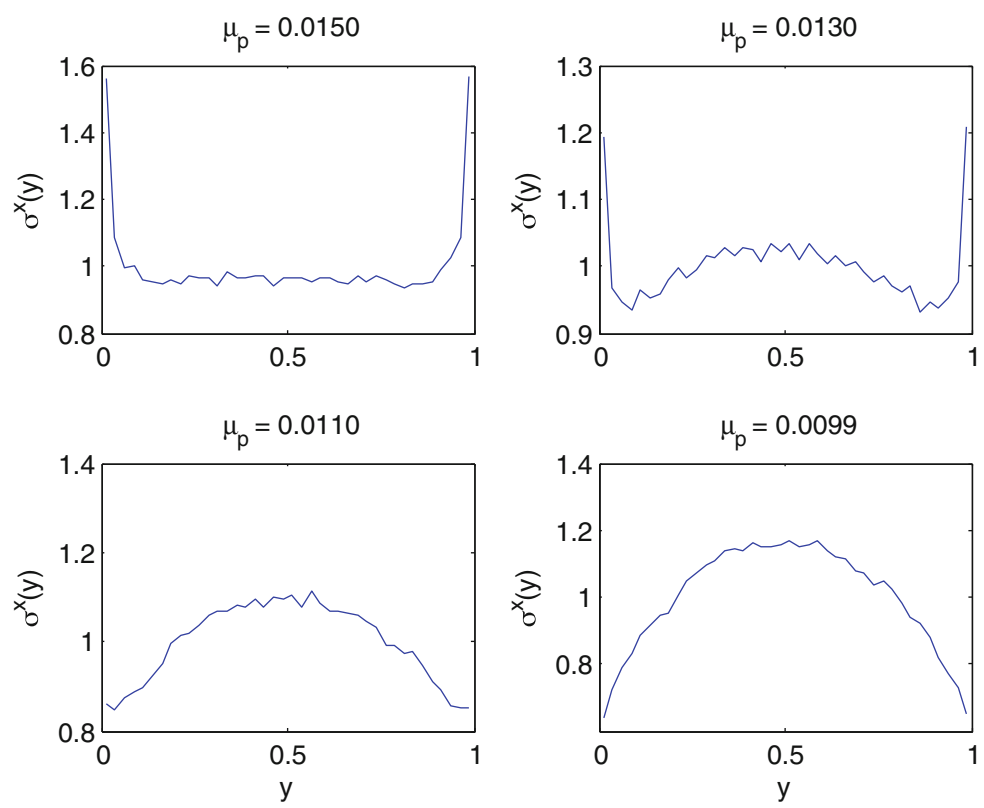

Fig. 3 The marginal density functions for protein $(y)$. The parameters are set as in Fig. 1 but $\mu_{p}$ is varied over a larger range. As $\mu_{p}$ nears $\lambda$, a mode appears at intermediate protein levels. The peaks at $y=0$ and $y=1$ disappear for low $\mu_{p}$

a Beta distribution exponentially fast. In particular, the unconditional distribution of expression levels is a Beta distribution scaled to the interval $\left(r_{0} / \mu, r_{1} / \mu\right)$ with parameters $\alpha=\lambda_{0} / \mu, \beta=\lambda_{1} / \mu$ (see Eq. 17).

Our analysis shows that the moments of the transient distribution approach those of the stationary distribution with an exponential rate, with a half life that depends on the maximum of $1 / \mu$ or $1 /\left(\lambda_{0}+\lambda_{1}\right)$. When $\mu$ is larger than $\lambda_{0}+\lambda_{1}$ this means that the decay towards the stationary moments has a half life of (ln $2 / 2$ times) the harmonic mean of the expected time in each environment. In either case the stationary distribution can be used to calculate functional aspects of organisms that persist for several oscillations of the environmental process.

These results can be directly used both to make predictions about experimental results and to calculate interesting statistics based on gene expression. In particular, the environment specific stationary distributions are relevant to understanding how closely gene expression tracks environmental change (Eq. 13). They represent the probability density of finding the system in both a particular environment and a particular level of expression. For example, $\pi_{0}(x)$ gives the probability of finding a cell in environment 0 at expression level $x$. In many instances, the ability of a cell to function, metabolize, and synthesize useful products depends on producing proteins in specific contexts. For instance, if the environmental switching process measures the availability of different sugars, then energy production will depend on the timing of the availability of specific enzymes. If fitness depends on total energy gain, then lifetime fitness can be calculated simply by taking the expectation of fitness over the 
stationary distribution, and our results on the convergence of statistics guarantee that this is a reasonable approximation if individuals persist over several fluctuations of the environment (the exact rate of convergence depends on $\mu$ ).

The distribution of expression levels can be interpreted in two different ways. First, it can be thought of as describing the dynamics of a single individual forward in time. In this interpretation, the PDF describes the probability density of finding the cell in at a particular expression level at a random time point far in the future. Second, it can be thought of as describing the dynamics of a population of cells that face uncorrelated random environments. In this case, it describes the frequency of cells that have a specific value of expression in the population.

This population level interpretation of the distribution of expression values is particularly useful because it can potentially be used to experimentally infer particular mechanisms of gene expression. There is much interest in reconstructing the topological structure of gene networks without individually assaying the physical interactions between signals and genes (Wagner 2001; Proulx et al. 2005). Population level measurements of protein variability are becoming feasible based on flow cytometry approaches (Newman et al. 2006). Our results show that genes under simple direct regulation of transcription rates will show Beta distributed expression levels when exposed to a randomly varying environment, while more complex forms of regulation will likely alter this pattern. This suggests that the distribution of expression levels could be used as a first pass filter to identify genes that do not have interactions with other genes, although further validation would be required.

We also developed an extended model that includes the additional step of protein production. So long as protein degradation rates are high relative to the rate of environmental change, the two-step mRNA $\rightarrow$ protein model shows similar dynamics to the one-step model of mRNA production. When protein turnover rates are lower, however, protein abundance can no longer track the environmental shifts. Because the protein concentration dynamics depend on the mRNA dynamics, any loss of environmental signal that occurs at the mRNA level is necessarily transferred to the protein level. Thus, high protein turnover rates cannot compensate for low mRNA turnover rates.

Our results highlight the importance of high turnover rates in allowing gene expression and protein abundances to track changes in the environment. We suggest that this has a relationship to an organisms ability to buffer noise in the environment (Proulx and Phillips 2005; de Visser et al. 2003). When individuals experience multiple environments over their lifespan, evolutionary theory predicts that selection will maximize the mean fitness of individuals and will not maintain multiple genotypes without genetic overdominance (Ellner 1996). Multicellular organisms can buffer changes in the external environment at the cellular level, so that the internal environment faced by cells may be relatively constant. Single celled organisms, on the other hand, are relatively exposed to changes in the environment and may be more dependent on gene regulation and intrinsic metabolic buffering to survive (Papp et al. 2004).

This leads to the prediction that high mRNA and protein turnover rates are required for single-celled organisms to maintain protein complements that track environmental change. Additionally, single celled organisms that experience relatively constant environments may be free to evolve lower turnover rates without a sacrifice in 
equilibrium protein abundance. It is known that gene length and structure affect the rate of transcription and mRNA stability (Feng and Niu 2007), but the range of values shows that turnover rates are generally higher in single celled organisms. For E. coli, the range of mRNA half-lives is as low as 1-2 min and up to $15 \mathrm{~min}$ (Feng and Niu 2007; Meyer et al. 2004), while in yeast the minimum half-lives are 2-3 min but can be as high as $90 \mathrm{~min}$ (Meyer et al. 2004). Mammals have higher minimum half-lives of around $15 \mathrm{~min}$, and the $\beta$-globin message has a half-life of more than 1 day (Meyer et al. 2004). Protein half-lives have been systematically measured in yeast, where they range from about 4 min to more than 10 hours (Belle et al. 2006).

While high degradation rates can allow a cell to track environmental shifts, they may entail significant costs. Wagner $(2005,2007)$ estimated the energetic costs of changing gene expression levels. He estimates that a change in equilibrium protein abundance of $0.5 \%$ for an average yeast gene would be visible to natural selection. Likewise, increasing protein turnover rates but maintaining a constant equilibrium would entail an energetic cost. This suggests that lower degradation rates will be favored by natural selection whenever the environment that individual cells face is relatively constant.

Open Access This article is distributed under the terms of the Creative Commons Attribution Noncommercial License which permits any noncommercial use, distribution, and reproduction in any medium, provided the original author(s) and source are credited.

\section{References}

Allen L (2003) An Introduction to stochastic processes with applications to biology. Prentice Hall, Upper Saddle River

Athreya K, Lahiri S (2006) Measure theory and probability theory. Springer, New York

Belle A, Tanay A, Bitincka L, Shamir R, O’Shea E (2006) Quantification of protein half-lives in the budding yeast proteome. Proc Nat Acad Sci 103(35):13004-13009

Bennett M, Pang WL, Ostroff N, Baumgartner B, Nayak S (2008) Metabolic gene regulation in a dynamically changing environment. Nature 454:1119-1122

Cook D, Gerber A, Tapscott S (1998) Modeling stochastic gene expression: Implications for haploinsufficiency. Proc Nat Acad Sci 95:15641-15646

Davis MHA (1984) Piecewise-deterministic markov processes: a general class of non-diffusion stochastic models. J Royal Stat Soc B 46:353-388

de Visser JAGM, Hermisson J, Wagner GP, Meyers LA, Bagheri-Chaichian H, Blanchard JL, Chao L, Cheverud JM, Elena SF, Fontana W, Gibson G, Hansen TF, Krakauer D, Lewontin RC, Ofria C, Rice SH, von Dassow G, Wagner A, Whitlock MC (2003) Perspective: evolution and detection of genetic robustness. Evolution 57(9):1959-1972

Dekel E, Mangan S, Alon U (2005) Environmental selection of the feed-forward loop circuit in gene-regulation networks. Phys Biol 2(2):81-88

Diaconis P, Freedman D (1999) Iterated random functions. SIAM Rev 41:45-76

Durrett R (2004) Essentials of stochatic processes. Springer, New York

Ellner SP (1996) You bet your life: life-history strategies in fluctuating environmnets. In: Othmer HG, Adler FR, Lewis MA, Dallon JC (eds) Case studies in mathematical modeling: ecology, physiology, and cell biology. Prentice Hall, Upper Saddle River, pp 3-24

Feng L, Niu DK (2007) Relationship between mRNA stability and length: an old question with a new twist. Biochem Genet 45(1-2):131-137. doi:10.1007/s10528-006-9059-5

Freund J (1971) Mathematical statistics, 2nd edn. Prentice Hall, Englewood Cliffs

Gander M, Mazza C, Rummler H (2007) Stochastic gene expression in switching environmnets. J Math Biol 55:249-269

Halbeisen RE, Galgano A, Scherrer T, Gerber AP (2008) Post-transcriptional gene regulation: from genome-wide studies to principles. Cell Mol Life Sci 65:798-813 
Horn R, Johnson C (1985) Matrix analysis. Cambridge University Press, New York

John F (1978) Partial differential equations, 3rd edn. Springer, New York

Karmakar R, Bose I (2004) Graded and binary responses in stochastic gene expression. Phys Biol 1:197-204

Karmakar R, Bose I (2007) Positive feedback, stochasticity and genetic competence. Phys Biol 4:29-37

Klyatskin VI (2005) Dynamics of stochastic systems. Elsevier, Amsterdam

Kussell E, Leibler S (2005) Phenotypic diversity, population growth, and information in fluctuating environments. Science 309:2075-2078

Mazza C, Piau D (2001) Dubins-freedman processes and rc filters. Ann Appl Probab 11:1330-1352

McAdams HH, Srinivasan B, Arkin AP (2004) The evolution of genetic regulatory systems in bacteria. Nat Rev Genet 5(3):169-178. doi:10.1038/nrg1292

McFadden J (1959) The probability density of the output of an rc filter when the input is a binary random process. IRE Trans IT-5:174-178

Meyer S, Temme C, Wahle E (2004) Messenger RNA turnover in eukaryotes: pathways and enzymes. Crit Rev Biochem Mol Biol 39(4):197-216. doi:10.1080/10409230490513991

Milstein G, Repin Y (1972) Action of Markov process on a system of differential equations. Differ Equ 5:1010-1019

Newman JRS, Ghaemmaghami S, Ihmels J, Breslow DK, Noble M, Derisi JL, Weissman JS (2006) Singlecell proteomic analysis of $S$. cerevisiae reveals the architecture of biological noise. Nature 441(7095): 840-846. doi:10.1038/nature04785

Papp B, Pál C, Hurst LD (2004) Metabolic network analysis of the causes and evolution of enzyme dispensability in yeast. Nature 429(6992):661-664. doi:10.1038/nature02636

Pawula R, Rice S (1986) On filtered binary processes. IEEE Trans Inf Theory IT-32:63-72

Pinsky M (1991) Lectures on random evolution. World Scientific, Singapore

Proulx SR, Promislow DE, Phillips PC (2005) Network thinking in ecology and evolution. Trends Ecol Evol 20(6):345-353. doi:10.1016/j.tree.2005.04.004

Proulx SR, Phillips PC (2005) The opportunity for canalization and the evolution of genetic networks. Am Nat 165(2):147-162. doi:10.1086/426873

Seshasayee A, Bertone P, Fraser G, Luscombe N (2006) Transcriptional regulatory networks in bacteria: from input signals to output responses. Curr Opin Microbiol 9(5):511-519. doi:10.1016/j.mib.2006. 08.007

Shahrezaei V, Swain PS (2008) Analytical distributions for stochastic gene expression. Proc Nat Acad Sci 105:17256-17261

Srinivasan S, Vasudevan R (1971) Introduction to random differential equations and their applications. Elsevier, New York

Wagner A (2001) How to reconstruct a large genetic network from $\mathrm{n}$ gene perturbations in fewer than $\mathrm{n}(2)$ easy steps. Bioinformatics 17(12):1183-1197

Wagner A (2005) Energy constraints on the evolution of gene expression. Mol Biol Evol 22(6):1365-1374. doi:10.1093/molbev/msi126

Wagner A (2007) Energy costs constrain the evolution of gene expression. J Exp Zool B Mol Dev Evol 308(3):322-324. doi:10.1002/jez.b.21152

Wittkopp P (2007) Variable gene expression in eukaryotes: a network perspective. J Exp Biol 210(9):15671575. doi: $10.1242 /$ jeb.002592 